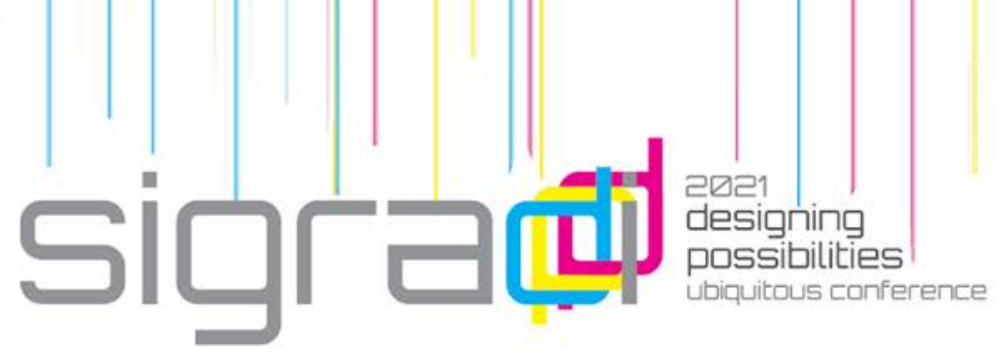

\title{
Codesign and Digital Fabrication: Applications in the Project Process with Visually Impaired People and Children
}

\author{
Luísa Fernanda Nercolino Deon, Priscila Castioni Isele, Alana Arena, Andrea \\ Quadrado Musssi \\ Faculdade Meridional - IMED, Brasil \\ luisa.deon@hotmail.com \\ priscila.castioni@hotmail.com \\ arquit.alana.arena@gmail.com \\ andrea.mussi@imed.edu.br
}

\begin{abstract}
The Co-design method has great relevance in the inclusion of different classes of users in the Design Process (DP), such as Visually Impaired People (PwDS) and children. This work indicates results of three Co-design dynamics. Focus Group, guided tour, semi-structured interviews, questionnaires and workshops were carried out, as strategies for the inclusion of different users in the DP. The process of designing an inclusive signage board for PwDS is presented. Next, the project of inclusive playgrounds for visually impaired children. Finally, an activity carried out with basic education students, combining new technologies in the child's learning process. Participants were encouraged to express their knowledge through different tools that adapted to their reality. The results show the importance of including different methods, tools and resources to support the DP. In addition, Digital Fabrication and Prototyping are techniques that enhance Co-design, as they act as congratulators for communication between the designer and users.
\end{abstract}

Keywords: Co-design, Digital Fabrication, Inclusion, Design Process, Collaborative Project.

\section{Introdução}

Nos últimos anos a tecnologia tornou-se uma das principais ferramentas de comunicação e informação, estando fortemente inseridas no cotidiano dos usuários. De acordo com Lawson (2011), esses movimentos tecnológicos impulsionam transformações em diferentes contextos, cenários e escalas. Pupo (2008) disserta sobre as novas ferramentas de apoio na forma de projetar 
em arquitetura decorrentes das novas tecnologias, tomando como exemplo as impressoras 3D e máquinas de corte a laser.

Os laboratórios de Fabricação Digital e Prototipagem voltados para o processo de projeto em arquitetura, tornam-se aliados no processo de construção de aprendizados (PUPO, 2008). Tais espaços representam a ressignificação dos processos em arquitetura, engenharia e design, expandindo novas possibilidades de criação e produção (BLIKSTEIN, 2013).

São espaços de Prototipagem estruturados com equipamentos tecnológicos, ferramentas de marcenaria, kits de robótica e eletrônica, dentre outros (EYCHENNE, NEVES, 2013; AGUSTINI, 2014; COSTA, PELEGRINI, 2017). Estão presente em ambientes educacionais de nível básico a nível superior, empresas privadas, como também, em espaços de lazer e convivência, como por exemplo museus e casas de cultura.

A metodologia mais válida na introdução de usuários nos processos de projeto de arquitetura, é a metodologia colaborativa, na qual os usuários participam efetivamente da elaboração do projeto, ajudando nas prováveis propostas a partir das suas experiências diárias sobre os locais (CAIXETA, FABRICIO, 2018). O objetivo é proporcionar melhor entendimento entre toda equipe envolvida, por meio de dinâmicas de comunicação, que é a chave do processo do Codesign aplicado na Arquitetura (MUSSI et al., 2019).

O Codesign é uma metodologia com foco na participação ativa dos usuários envolvidos, com o objetivo de compreender suas habilidades, competências e conhecimentos, sobre a temática em estudo (SANDERS, STAPPERS, 2008). Refere-se a uma técnica de projeto realizada junto com o usuário e não apenas para sua utilização (MAGNUSSON et al, 2018; MUSSI et al., 2019; MUSSI et al., 2020). Além disso, estudos apontam que o Codesign aliado as novas tecnologias de Fabricação Digital e Prototipagem, podem ser aplicados como ferramentas na construção de conteúdos pedagógicos em instituições de ensino de nível básico a nível superior (ANGELO, 2020; BLIKSTEIN, 2016).

Desta forma, este trabalho tem como objetivo indicar métodos e ferramentas de Codesign com o uso da Fabricação Digital e Prototipagem, que auxiliam o arquiteto no processo de projeto de espaço internos, bem como, as possibilidades de uso na construção de conteúdo pedagógico, aliados a metodologias ativas na educação básica.

O estudo relata três dinâmicas com aplicabilidade do Codesign como fonte de comunicação com os usuários (adultos com deficiência visual, crianças com e sem deficiência visual e, professoras da educação básica), utilizando-se das técnicas de Fabricação Digital e Prototipagem para sua condução. Além disso, como suporte ao método de Codesign também foram utilizados Focus Group (FG), passeio acompanhado, entrevistas semiestruturadas, questionários e workshops. 


\section{Prototipagem: Suporte ao Codesign no projeto}

2 Métodos e Ferramentas de Fabricação Digital e arquitetônico

As metodologias mais utilizadas no processo de Codesign na Arquitetura são: informativas, na qual os usuários apenas compartilham ou recebem as informações; consultivas onde os usuários são submetidos a conversações sobre os serviços do projeto; e as colaborativas, em que os mesmos acompanham efetivamente o processo de projeto (CAIXETA, FABRICIO, 2018).

\subsection{Fabricação Digital como Ferramenta de Codesign para confecção de uma Placa Sinalética Inclusiva para pessoas com deficiência visual.}

O trabalho teve como objetivo indicar exemplos de aplicabilidade do Codesign, por meio da criação de um produto que represente em escala reduzida ambientes internos reais, com o intuito de melhorar a mobilidade e autonomia das pessoas com deficiência visual (PcDV). O problema em questão foi apresentar de forma multisensorial às PCDV como estaria organizado o ambiente em que estes usuários estariam acessando, por meio de uma Placa Sinalética, que ficaria junto às portas de entradas dos ambientes e sinalizaria de forma multissensorial a localização das paredes, portas e obstáculos que eles poderiam encontrar.

O processo de projeto da Placa Sinalética passou por três etapas. Os usuários participaram do processo de escolhas, adaptação e melhorias do produto. Inicialmente testou-se formas de representação e sinalização do ambiente em estudo, neste caso, um laboratório de metodologias inovadoras, no qual comporta intensa alternância de layouts interno. Para representação do ambiente foi utilizada chapas de MDF $6 \mathrm{~mm}$ e $3 \mathrm{~mm}$; e palitos de bambu. $O$ modelo foi confeccionado na cortadora à laser, em formato de malha com pontos representados por palitos de bambu de $4 \mathrm{~mm} \times 4 \mathrm{~mm}$ que sinalizava um quadrante de $50 \mathrm{~cm} \times 50 \mathrm{~cm}$ no ambiente real (Figura 1).
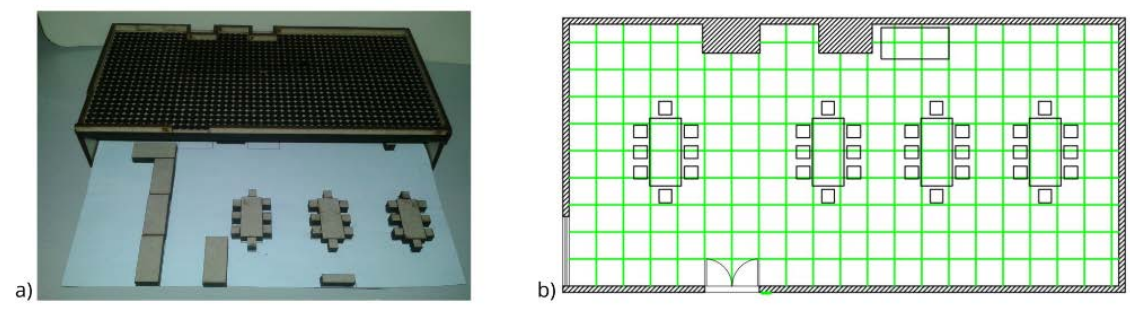

Figura 1. (a) Placa sinalética etapa 1 (b) Representação da malha no ambiente real. Fonte: Autores, 2020. 


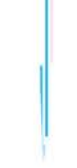

Posteriormente, seis pessoas com deficiência visual participaram de um Workshop, que visou testar a memorização espacial e percepção por meio do toque dos usuários na Placa Sinalética. Os usuários tocavam na Placa antes de acessarem o local representado, na sequência eram instigados a encontrar um objeto no ambiente (Figura 2). Por meio da aplicação de um questionário, foi possível compreender diversas percepções dos participantes, contribuindo para a segunda etapa de melhoria do produto.

a)
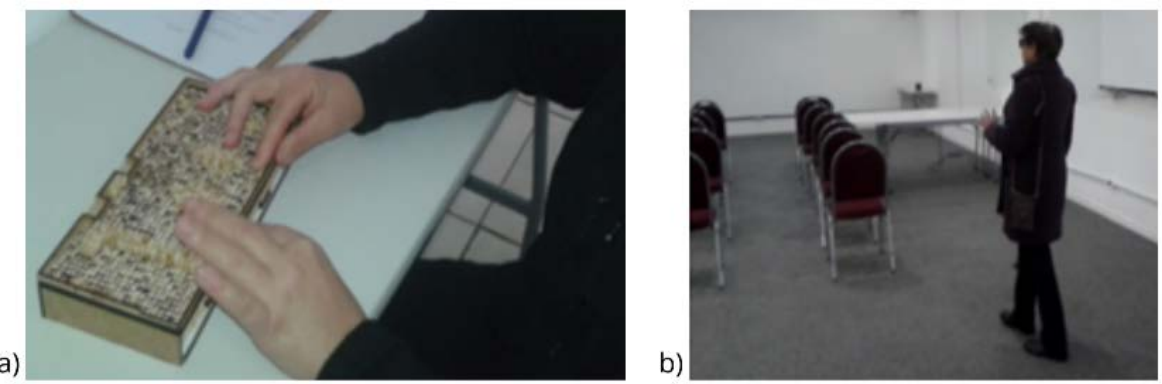

Figura 2. (a) Participante tateando a Placa (b) Passeio na sala. Fonte: Autores, 2020.

Para segunda etapa do projeto considerou-se algumas alterações, como por exemplo: alteração do material utilizado, passando de MDF para o PLA rígido na cor branca; aumento de escala de desenho e inserção de tecnologia eletrônica com o uso do micro motor vibratório.

$\mathrm{Na}$ sequência os usuários avaliaram as modificações, considerando principalmente o material, textura, formato e tecnologia inserida, para dar seguimento a próxima etapa do projeto, que visa a produção em maior escala. A metodologia utilizada foi o Teste A/ B onde avalia-se por parte do usuário uma escolha em duas opções disponíveis em diversos critérios (MARTIN, HANINGTON, 2012).

Considerando as percepções dos participantes, para a terceira etapa as modificações da Placa Sinalética foram: alteração do tipo de componente eletrônico inserido (micro motor vibratório por um eletroímã solenóide), e consequentemente, alterou-se o formato do produto. Além disso, substitui-se as cores do PLA para obter contraste para que os usuários com baixa visão pudessem ter mais percepção visual do produto (Figura 3). 


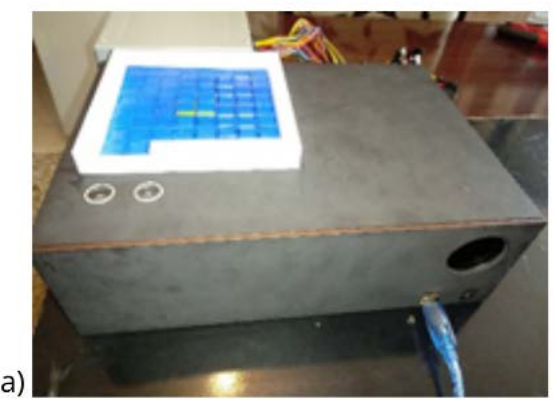

Figura 3. (a) Placa sinalética etapa 3. (b) Fiação dos componentes eletrônicos.

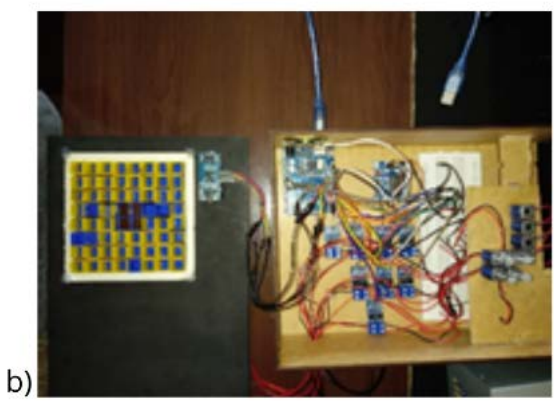

b) Fonte: Autores, 2020.

\subsection{Fabricação Digital como Ferramenta de Codesign no Projeto de Playgrounds Inclusivos.}

O principal objetivo deste trabalho foi explorar novos métodos, técnicas e ferramentas de Codesign no projeto de paisagismo, incluindo Pessoas com Deficiência Visual (PcDV) no PP, bem como estabelecer novas características para os processos e produtos em espaços de lazer.

O local de estudo de caso para o projeto de pesquisa foi a Praça Mário Bernardi que fica localizada em frente à escola, e contou com a participação de dez crianças com idades de 6 à 12 anos estudantes da Escola Frei Wilson João, sendo 4 alunos com baixa visão.

Inicialmente confeccionou-se duas maquetes táteis, com objetivo de facilitar a comunicação entre os projetistas e os participantes. A primeira maquete refere-se ao playground já existente na escola, do qual os participantes já estão familiarizados. A segunda maquete é referente ao playground existente na Praça.

As maquetes foram projetadas inicialmente pelo software Revit (2021) e após modeladas no software TinkerCad (2021). Na sequência, foram fabricadas digitalmente, utilizando a impressora 3D (Figura 4).
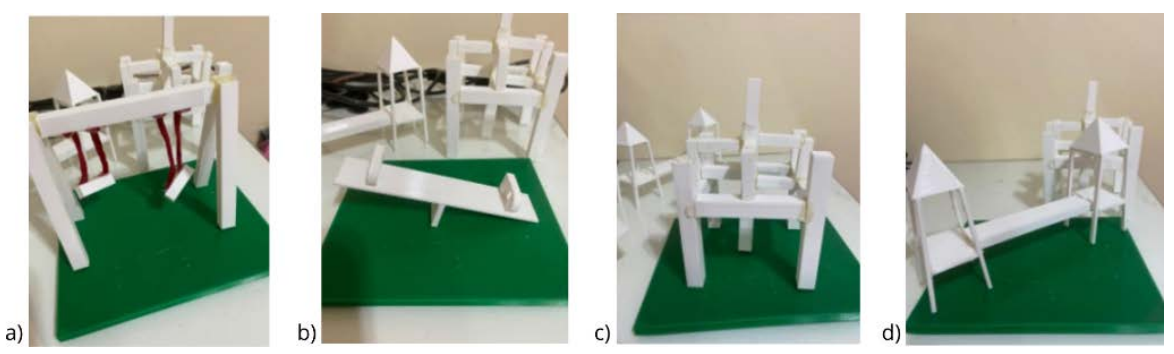

Figura 4. Maquetes táteis do playground da escola: (a) balanço (b) gangorra (c) trepa-trepa (d) maquete tátil do playground da praça. Fonte: Autores, 2020. 
A dinâmica ocorreu de forma online. As maquetes foram disponibilizadas aos participantes para manipulação em casa, respeitando o cronograma proposto para a articulação da atividade. Posteriormente realizou-se um Focus Group (FG) para debate e compreensão das percepções das crianças em relação as características dos playgrounds. Por fim, realizou-se entrevista semiestruturada.

Durante a manipulação da primeira maquete, oito dos dez participantes reconheceram os brinquedos presentes no playground da escola, mencionando já terem brincado em tais equipamentos. Os alunos manifestaram entusiasmo em vivenciar essa nova experiência de tatear a maquete de um ambiente presente no seu cotidiano. A maquete do playground em menor escala, instigou as crianças a curiosidade em saber sobre seu processo de montagem.

Os pais dos alunos participantes discorreram sobre alguns aspectos do playground da escola, dentre eles a materialidade dos brinquedos e segurança. Além disso, consideraram o balanço do playground como o brinquedo mais interativo e inclusivo.

Já na manipulação da maquete do playground da Praça, algumas crianças abordaram já terem feito o uso do local, mas ao tatear perceberam a falta do escorregador e das escadas. Para segurança dos participantes o escorregador e as escadas não foram mantidos na maquete, pois apresentaram rebarbas durante seu processo de confecção.

$\mathrm{Na}$ articulação do $F G$, as crianças abordaram as diferenças entre as maquetes. Mencionaram ser divertido e interessante a presença de diferentes brinquedos, como por exemplo o playground da escola, que comporta uma diversificada gama de equipamentos, diferente do playground da Praça, que contém apenas um módulo interativo.

$\mathrm{Na}$ última etapa do $F G$ questionou-se a percepção das crianças em utilizar as maquetes. Todas apontaram ter gostado da experiência, e indicaram que gostariam de fazer uso de maquetes táteis como meio de comunicação para realização de projetos. Além disso, apontaram importância de integrar crianças no PP de espaços infantis, acolhendo suas percepções e contribuições.

\subsection{Fábrica de Brinquedos do Papai Noel}

A atividade foi elaborada em duas edições e contou com um total de 40 estudantes com faixa etária de 4 a 8 anos, e teve como principal objetivo o aprendizado por meio da troca de experiências entre discentes de escola privada e instituição pública, por meio da construção de brinquedos educativos.

As crianças foram divididas em grupos com no máximo 5 componentes por mediador. Cada estação de trabalho reunia um conjunto de material de apoio composto por um passo a passo de montagem, um modelo de cada brinquedo pronto para análise. Na sequência foi distribuído um kit com peças em MDF e canetas hidrocor para iniciar a fabricação e personalização dos objetos, sendo esses um ônibus, uma catapulta e um pião (Figura 5). 

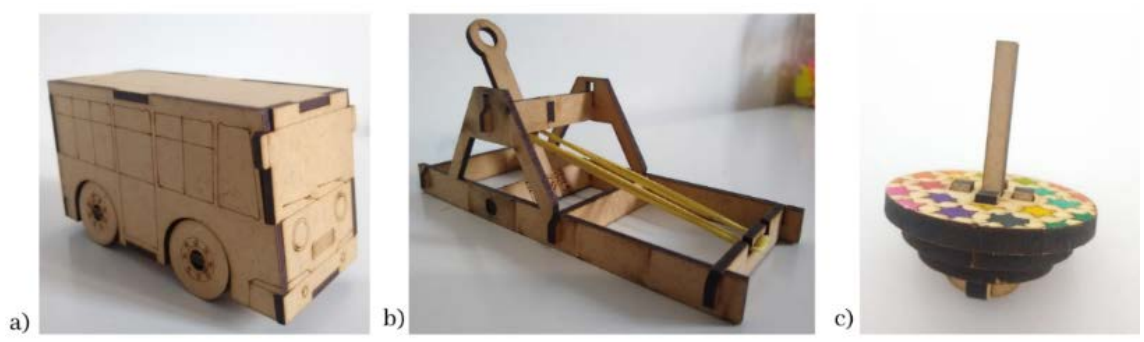

Figura 5. (a) ônibus (b) catapulta (c) pião. Fonte: Autores, 2019.

Durante a montagem os alunos eram instigados a resolução de alguns problemas, por meio de questionamentos realizados pelas mediadoras, como por exemplo: Como é o atrito do ônibus em relação ao conjunto roda e eixo? $\mathrm{O}$ que acontece se o eixo de seção retangular for substituído pelo eixo de seção circular? Como é o desempenho das rodas nos diferentes tipos de solos? Da mesma maneira, a capacidade de impulsão da Catapulta resultante da tensão e força depositada no elástico foi apresentada aos alunos, com a pergunta de como o material lançado pode alcançar uma distância maior? Logo, a personalização do pião com canetas hidrocor coloridas, viabilizou o entendimento de: Quais cores complementares podem obter a partir da rotação do brinquedo no solo? E quais as outras possibilidades de cores complementares podem ser geradas?

Toda essa gama de aprendizado norteada pela metodologia aker, despertou curiosidades relacionadas aos objetos montados. Os alunos queriam saber como eram fabricadas as peças que originavam tais brinquedos. Dessa forma, a turma foi dividida em dois grupos nos quais eram convidados a visitar o espaço onde se encontrava a cortadora a laser, equipamento utilizado para confecção das peças em MDF.

\section{Resultados e Discussões}

A primeira dinâmica referente a utilização de Codesign no processo de projeto de uma Placa Sinalética Inclusiva, inseriu usuários com deficiência visual em todas as etapas de criação e avaliação do produto proposto, acolhendo suas percepções. O uso da cortadora a laser e impressora 3D na confecção do produto, proporcionou a comunicação entre usuários e projetistas, fornecendo importantes reflexões referente aos diferentes equipamentos e materiais utilizados, até chegar ao produto final (Figura 6). 

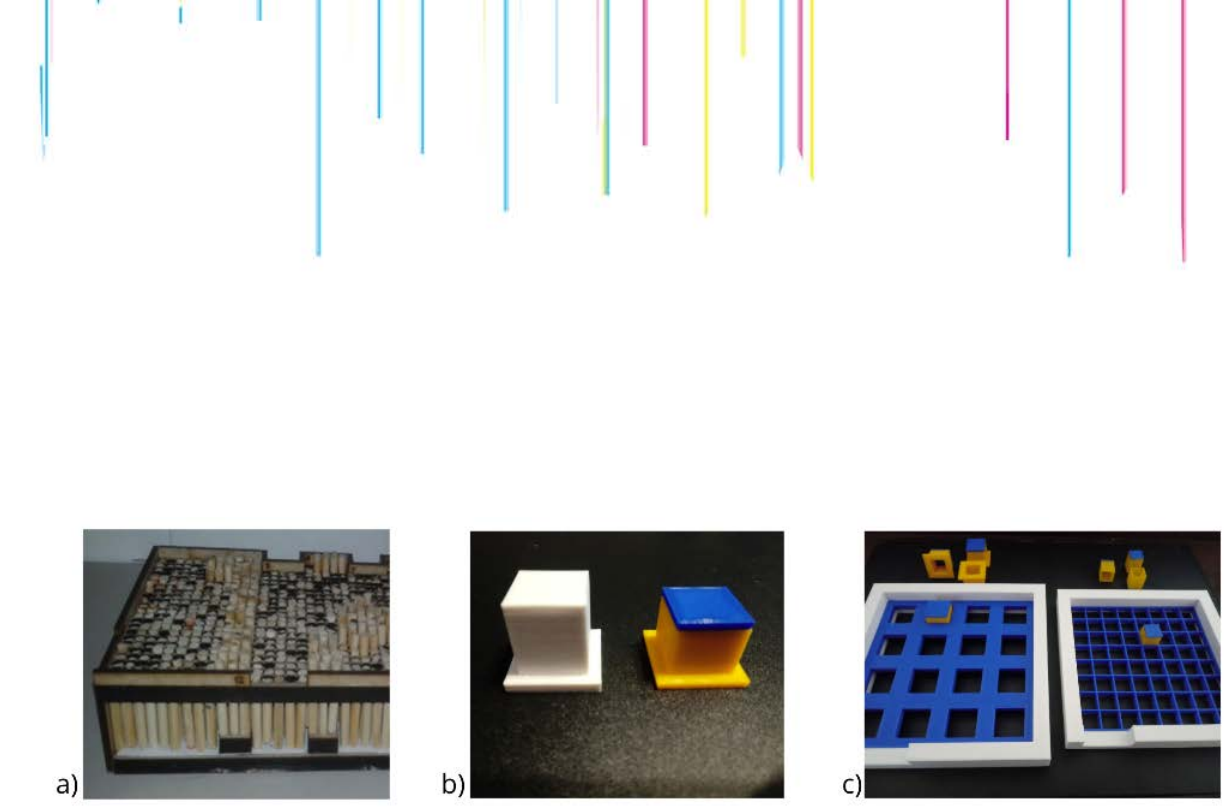

Figura 6. (a) Textura em madeira (b) Alteração de dimensões e cores (c) Alteração de espessura de material PLA. Fonte: Autores, 2020.

A substituição das chapas de MDF pelo PLA rígido, ocorreu a partir da compreensão de que para os usuários. Quanto mais liso o material, melhor, para não confundir com outras elevações que teriam importância de informação.

Outro aspecto importante foi a utilização do contraste de cores, ou seja, a importância em fazer uso das seguintes combinações de cores: branco e preto ou azul e amarelo, para pessoas com baixa visão.

Referente ao teste dos componentes eletrônicos na etapa 2, o uso do vibracall gerou dúvidas na questão de sensibilidade por parte dos usuários, pois não havia pontualidade de informação. A vibração gerada pelos micromotores, gerava um campo vibracional que interferia nos pontos em que não poderiam vibrar. Desta forma, foi necessário trocar o material ou encontrar um outro componente eletrônico que realizasse a sinalização pontual. Escolheu-se como componente o Eletroímã Solenóide, o qual obteve êxito na sinalização da placa por parte dos usuários.

$\mathrm{Na}$ etapa 3 inseriu-se outros componentes eletrônicos, tomando como exemplo o sensor de presença, gravador com autofalante e programação em Arduino. Tais componentes realizavam a detecção da presença do usuário em frente a Placa e pronunciavam uma gravação falada de informações referente a Placa.

Enquanto que a segunda dinâmica de projeto de paisagismo em playgrounds infantis, possibilitou explorar novos métodos, ferramentas e recursos de Codesign no PP de paisagismo, por meio de estratégias de projeto participativo. Além disso, gerou engajamento e autonomia na inclusão de PcDV.

A manipulação das duas maquetes táteis ocorreu de forma livre (Figura 7). As crianças integraram seus brinquedos durante o processo de exploração da maquete. Geraram outras tipologias de interação no processo criativo. 
Em relação ao material proposto, o MDF, foi percebido como envolventes para a construção dos objetos, bem como, promoveu a curiosidade em relação a confecção das peças. A customização dos brinquedos com o uso de canetas hidrocor, foram essenciais para criar identidade e autonomia no processo criativo.

Embora esta atividade tenha promovido a construção de brinquedos que estão no imaginário das crianças, percebe-se que há possibilidades de explorar de forma mais aprofundada o contexto de mundo real durante as dinâmicas. Com isso, indica-se como potencial para atividades futuras, apropriar-se de jogos e brincadeiras presentes no cotidiano dos alunos e exercitar o Codesign na criação de novos brinquedos e interações com eles e entre os participantes no aprendizado.

\section{Conclusão}

Este trabalho apresentou três formas de aplicabilidade do método de Codesign, tomando partido do uso da Fabricação Digital e Prototipagem, como veículo de comunicação entre usuários e projetistas. Incluiu-se usuários em todas as etapas do processo de projeto, acolhendo suas percepções para tomadas de decisões.

Compreende-se que durante o processo de aplicação do método de Codesign, os participantes são incentivados a expressar seus conhecimentos, por meio diferentes ferramentas que se adaptam a sua realidade. Além disso o trabalho mostrou que a diversificada gama de mecanismos para condução do projeto permite ampliar a criatividade tanto dos usuários, como dos arquitetos, criando novas percepções e possibilidades projetais.

Tanto na primeira dinâmica de projeto da Placa sinalética, como na segunda dinâmica de projeto de playground inclusivo, foi possível observar a importância da tipologia de cores na tatilidade dos objetos. Compreende-se que é necessário utilizar cores contrastantes em maquetes táteis para pessoas com baixa visão.

Já a segunda dinâmica resultou na construção de diretrizes metodológicas para projetos de playground infantis, uma vez que, não havia relatos de aplicabilidade de tal técnica, principalmente, com foco em crianças com deficiências visual, e ainda, utilizando maquetes táteis confeccionadas com técnicas de Fabricação Digital e Prototipagem.

$\mathrm{Na}$ condução da terceira dinâmica, compreendeu-se que os materiais, ferramentas e mecanismos presentes no cotidiano de profissionais e estudantes de arquitetura, podem ser adaptados e direcionados para construção de materiais e conteúdos pedagógicos, mais especificamente para educação básica, tomando como por exemplo as habilidades de modelagem 2D e 3D, Fabricação Digital, Prototipagem, criação de conceitos, emprego da materialidade, entre outros. 
Importante ressaltar que esses elementos não são tratados, como forma de exclusão das técnicas ou materiais tradicionais de ensino, mas sim, objetiva unir materiais comumente encontrados no ambiente escolar a elementos tecnológicos, considerando sobretudo o pleno desenvolvimento do aluno.

Por fim, conclui-se a que o Codesign é um método que pode ser aplicado em diferentes, contextos, escalas e cenários. Além disso a Fabricação Digital e Prototipagem são potenciais veículos de comunicação para articulação desse processo.

Agradecemos a Associação Passofundense de Cegos - APACE, as Escolas participantes e os entrevistados pela importante contribuição científica a este trabalho de pesquisa; a Fundação IMED; o Núcleo de Inovação e Tecnologia em Arquitetura e Urbanismo (NITAU) do Programa de Pósgraduação em Arquitetura e Urbanismo da IMED (PPGARQ/IMED); o Conselho Nacional de Desenvolvimento Científico e Tecnológico - CNPq; e a Fundação de Amparo à Pesquisa do Rio Grande do Sul - FAPERGS. O presente trabalho foi realizado em parte com apoio da Coordenação de Aperfeiçoamento de Pessoal de Nível Superior - Brasil (CAPES) - Código de Financiamento 001.

\section{Referências}

Agustini, Gabriela. (2014). O momento dos laboratórios como espaços de criatividade, ino-vação e invenção. In: Costa, Elaine; Agustini, Gabriela. De baixo para cima. Rio de Janeiro: Aeroplano, 192-218.

Angelo, Alex Garcia Smith. (2015). Considerações sobre um campo conceitua comum entre a formação básica escolar, projeto e as tecnologias de modelagem e fabricação. Disponível em: https://www.teses.usp.br/teses/disponiveis/16/16134/tde-07032016155459/publico/alexgarciarev.pdf

Blikstein, Paulo. (2013). Digital fabrication and 'making' in education: The democratization of invention. In: Walter-Herrmann, Julia; Büching, Corinne. FabLabs: Of ma-chines, makers and inventors. Bielefeld: Transcript Publishers, 203-221.

Blikstein, Paulo. (2016). Viagens em Troia com Freire: a tecnologia como um agente de emancipação. Educação Pesquisa. São Paulo. v. 42, (n. 3) https://doi.org/10.1590/S1517-970220164203003

Caixeta, Michele C. B. Ferrari; Fabricio, Márcio M. (2018). Métodos e in-strumentos de apoio ao codesign no processo de projeto. Ambiente Construído, Porto Alegre, v. 18, (n. 1), 111-131. https://doi.org/10.1590/s1678-86212018000100212

Celani, Gabriela; Sedrez, Maycon; Lenz, Daniel e Macedo, Alessandra. (2015). The Future of the Architects's Employment: To Which Extent Can Architectural Design be Computerised? In: Celani, Gabriela; Sperling, David Moreno; Franco, Juarez Moara Santos (Eds.). Computer-Aided Architectural Design, CAAD Futures. São Paulo, Brasil, v. 14, 195-212. 
Costa, Cristiane O.; Pelegrini, Alexandre V. (2020). O design dos Makerspaces e dos Fab Labs no Brasil: um mapeamento preliminar. Design e Tecnologia, v.7, (n. 13), 57-66. https://doi.org/10.23972/det2017iss13pp57-66

Eychenne, Fabien; Neves, Heloisa. (2013). Fab Lab: A vanguarda da Nova Revolução In-dustrial. São Paulo: Editorial Fab Lab Brasil. E-book. Disponível em https://livrofablab.wordpress.com/2013/08/05/pdf-free-download/

Lawson, Bryan. (2011). Como arquitetos e Designers pensam. Trad. Mari Beatriz. Medina. São Paulo: Oficinas de Textos.

Magnusson, Charlotte; Hedvall, Per-Olof; Caltenco, Héctor. (2018). Co-designing together with Persons with Visual Impairments. In: Pissaloux, Edwige; Velázquez, Ramiro. Mobility of Visually Impaired People: Fundamentals and ICT Assistive Technologies. Berlim: Springer. (pp. 411-434).

Martin, Bella; Hanington, Bruce. (2012). Universal Methods os Design: 100 ways to research complex problems, desevop, inoovative ideas, and design effective solutions. EUA: Rockport Publishers.

Mussi, Andréa Q.; Silva, Thaisa L.; Zardo, Paola; Silva, Juliano L.; Pazini, Ernani Z.; Ferri, Mariana; Moreira, Denise (2019). Ferramentas de incremento do bem-estar de pessoas com deficiência visual: arquitetura inclusiva e maquete tátil. Arquitetura revista (UNISINOS), v. 15, (pp. 1-14).

Mussi, Andréa Q.; Silva, Luisa B. O.; Lantelme, Elvira M. V.; Cesaro, Sara R.; Deon, Luisa F. N.; Rodrigues, Daiara I. ; SILVA, Thaisa L. (2020). Arquitetura inclusiva: experiência de projeto colaborativo. Ambiente Construído (ONLINE), v. 20, (pp. 367-386).

Peluzio, Bárbara C. (2017). Pocket Park: Projeto de Espaço Público no centro de Vila Velha/ES. Trabalho de Conclusão de Curso - Faculdade de Arquitetura e Urbanismo, Universidade de Vila Velha, Espirito Santo, p. 108. https://issuu.com/barbarachalhub/docs/pocket_park_b_rbara_chalhub_peluzi.

Pupo, Regiane. (2008). Ensino da prototipagem rápida e fabricação digital para arquitetura e construção no Brasil: definições e estado da arte. PARC Pesquisa em Arquitetura e Construção. Campinas: FEC UNICAMP. DOI: https://doi.org/10.20396/parc.v1i3.8634511

Revit. (2021). Disponível em: <https://www.autodesk.com.br/products/revit/overview>

Sanders, Elizabeth B.-N.; Stappers, Pieter Jan. (2018). Co-creation and the new landscapes of design. Co-design, v. 4, (n. 1), 5-18.

TinkerCad. (2021). Disponível em: https://www.tinkercad.com/

Willis, Dan; Woodward, Todd. (2010). Diminishing difficulty: mass customization and the digital production of architecture. In: Corser, Robert. (ed.) Fabricating architecture. New York: Princeton Architectural Press, 178-208. 